\title{
Long Noncoding RNA H19 Overexpression Protects against Hypoxic-Ischemic Brain Damage by Inhibiting miR-107 and Up-Regulating Vascular Endothelial Growth Factor

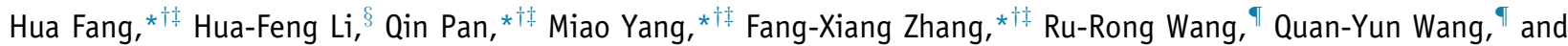
Jian-Ping Zhang ${ }^{\star \dagger \ddagger}$

From the Department of Anesthesiology, ${ }^{*}$ Guizhou Provincial People’s Hospital, Guiyang; the Department of Anesthesiology, ${ }^{\dagger}$ Guizhou University People's Hospital, Guiyang; the Laboratory of Anesthesiology and Perioperative Medicine, ${ }^{\ddagger}$ Guizhou University School of Medicine, Guiyang; and the Department of Anesthesiology, ${ }^{\S}$ West China Second University Hospital, and the Department of Anesthesiology, "West China Hospital, Sichuan University, Chengdu, China

\author{
Accepted for publication \\ November 16, 2020. \\ Address correspondence to \\ Jian-Ping Zhang, M.D., \\ Department of Anesthesiology, \\ Guizhou Provincial People's \\ Hospital, No. 83, Zhongshan E. \\ Rd., Guiyang 550002, Guizhou \\ Province, China. E-mail: \\ albatrossa@yeah.net.
}

\begin{abstract}
Long noncoding RNAs play critical roles in cellular homeostasis, and long noncoding RNA H19 (H19) is implicated in several pathologic conditions. The putative role of $\mathrm{H} 19$ in the pathogenesis and progression of hypoxic-ischemic brain damage (HIBD) is not yet understood. Therefore, a series of in vivo and in vitro experiments were designed to investigate the potential roles of $\mathrm{H} 19$ in neuronal apoptosis and cognitive dysfunction in HIBD. H19 expression was decreased in HIBD rat models established by partial occlusion of carotid artery. $\mathrm{H} 19$ bound to and decreased the expression of miR-107, which also increased VEGF expression. $\mathrm{H} 19$ overexpression reduced neuronal apoptosis and alleviated cognitive dysfunction in HIBD rats. The up-regulation of miR-107 reversed the protective effects conferred by H19. In addition, the cell model of HIBD was established by oxygen-glucose deprivation in neuronal cells used. H19 overexpression in oxygen-glucose deprivation neurons increased B-cell lymphoma-2 and decreased B-cell lymphoma-2-associated X, total and cleaved caspase-3 expressions. Taken together, the results showed that $\mathrm{H} 19$ expresses at a low level in HIBD. H19 overexpression decreased miR-107 and increased VEGF expression, which resulted in repressed neuronal apoptosis and alleviated cognitive dysfunction. Thus, H19 may serve as a molecular target for translational research for HIBD therapy. (Am J Pathol 2021, 191: 503-514; https://doi.org/10.1016/j.ajpath.2020.11.014)
\end{abstract}

Hypoxic-ischemic brain damage (HIBD) affects approximately $0.6 \%$ of neonates and $25 \%$ to $30 \%$ of newborns, with serious long-term consequences ${ }^{1}$ such as epilepsy, mental retardation, and cerebral palsy. ${ }^{2}$ HIBD is characterized by neuronal apoptosis, cognitive dysfunction, and damage to the hippocampus. ${ }^{3,4}$ At present, there is a notable scarcity of effective therapeutic strategies for HIBD. ${ }^{5}$ An understanding of the molecular events underpinning neuronal apoptosis is valuable for therapeutic advances for alleviation of HIBD.

Long noncoding RNAs (lncRNAs) have been implicated in the neurodevelopment and etiological pathogenesis of HIBD. ${ }^{6}$ LncRNAs represent RNA transcripts $>200$ nucleotides and comprise the largest portion of the transcriptome. ${ }^{7}$ LncRNAs play crucial roles in embryogenesis and development of the central nervous system. ${ }^{7}$ LncRNAs are highly expressed in the brain and have been reported to regulate several brain activities, including plasticity, homeostasis, and development. ${ }^{8}$ LncRNA H19 (H19) is the first identified lncRNA and has been

Supported by the National Natural Science Foundation of China number 81960239 (H.F.) and number 82060244 (J.-P.Z.), the Foundation of Science and Technology Department of Guizhou Province number GZSYQCC(2014)004 (H.F.), the Foundation of Science and Technology of Guizhou Province Health Commission number gzwjkj2020-1-133 (J.-P.Z.), and the Traditional Chinese-Ethnic Medicine Science and Technology Project of Guizhou Administration of Traditional Chinese Medicine QZYY-2020-002 (J.-P.Z.).

H.F. and H.-F.L. contributed equally to this work.

Disclosures: None declared. 
implicated in multiple pathologic conditions. ${ }^{9}$ The potential role of H19 in the pathogenesis and progression of HIBD is yet to be comprehensively described.

Certain IncRNAs can bind to miRNAs and diminish the bioavailability of miRNAs to their target mRNAs. ${ }^{10}$ miRNAs represent small noncoding RNAs of approximately $22 \mathrm{bp}$ in length that silence genes by degrading mRNAs or inhibiting translation. ${ }^{11}$ miRNAs are known to be critical modulators of post-transcriptional gene silencing in the development and pathogenesis of HIBD. ${ }^{12}$ Specifically, the activation of H19 was reported to suppress miR-107 expression and thus promote cell cycle progression of non-small-cell lung cancer, ${ }^{13}$ suggesting a binding site between H19 and miR-107. miR107 has been documented to play a vital role in the pathogenesis of traumatic brain injury and neurodegenerative disease, such as Alzheimer disease. ${ }^{14}$ The bioinformatics website Targetscan (http://www.targetscan.org/vert_71, last accessed December 14, 2018) adopted in our study to predict targeting relationship in HIBD, identified a specific binding site between the $3^{\prime}$-untranslated region (UTR) and miR-107 sequence, suggesting that $\mathrm{H} 19$ can target miR107. Moreover, evidence has been collected on the basis of bioinformatics analyses, indicating that vascular endothelial growth factor $(V E G F)$ is one of the target genes of miR-107. Expression of $V E G F$, a trophic factor, increases after central nervous system injury, promoting angiogenesis and neurogenesis after cerebral ischemia. ${ }^{15}$ Therefore, VEGF is regarded as a target molecule for ischemic brain injury. ${ }^{16} \mathrm{On}$ the basis of literature analysis, we hypothesized that HIBD results in decreased $\mathrm{H} 19$, increased miR-107, and decreased $V E G F$, which contribute to histologic and behavioral deficits.

\section{Materials and Methods}

\section{Ethics Statement}

Animal experimental protocols were approved by the Ethics Committee of Guizhou Provincial People's Hospital (approval number 201803005). All animal experiments were performed in accordance with the Guide for the Care and Use of Laboratory Animals, published by the US $\mathrm{NIH},{ }^{17}$ and care was taken to minimize the numbers and suffering of the included animals.

\section{Establishment of HIBD Rat Models}

Neonatal male Sprague-Dawley rats $(n=25$; age, 7 days; weight, $11.84 \pm 1.46 \mathrm{~g}$ ) were included in this study. The

Table 1 The Classification of Rats

\begin{tabular}{lll}
\hline Grouping & $\begin{array}{l}\text { Rats in } \\
\text { the experiment, } n\end{array}$ & $\begin{array}{l}\text { Successfully } \\
\text { modeled rats, } n\end{array}$ \\
\hline Sham-operated rats & 10 & 10 \\
HIBD modeled rats & 15 & 10 \\
\hline
\end{tabular}

HIBD, hypoxic-ischemic brain damage. rats were kept and breastfed under controlled conditions in an animal room with $20^{\circ} \mathrm{C}$ to $24^{\circ} \mathrm{C}$ temperature, $40 \%$ to $70 \%$ relative humidity, and $>50-\mathrm{dB}$ noise level in a 12 -hour light/dark cycle. A total of 15 rats were used to establish the HIBD model. The rats were anesthetized with an i.p. injection of $30 \mathrm{mg} / \mathrm{kg} 0.3 \%$ pentobarbital sodium solution. The rats were then placed onto an operating table in a supine position. The neck region was disinfected with alcohol, after which a $0.5-\mathrm{cm}$ long incision was made along the midline under the guidance of a dissecting microscope. The anterior cervical muscles and trachea were separated to expose the carotid triangle using a glass needle. Carotid pulsations were observed on the right side of the trachea and adjacent to the vagus nerve. The carotid artery was separated, after which the right cervical vessels were ligated with double partial occlusion to limit blood flow. The surgery lasted for a period of $<5$ minutes to minimize nonsurgical injuries from prolonged operation. The incision was closed using sutures. The rats were then placed in a cage for 1 to 2 hours following surgery. When the rats were confirmed to be in a fully awake state, they were placed in a sealed $50-\mathrm{mL}$ hypoxic container at $20^{\circ} \mathrm{C}$. After 2 hours of exposure to hypoxic conditions, the neonatal rats were breastfed. The remaining 10 rats were subjected to a sham operation, with all identical procedures conducted as on the HIBD rats, except for right carotid artery ligation (Table 1). The rats that fulfilled the following criteria were considered successful HIBD models: negative geotaxis (ie, response when rats were placed head down on an incline of 40 to 45 degrees); bar holding; finger climbing (ie, the ability to climb four horizontally extended fingers with the examiner's palm inclined at 60 degrees); and response score $<3$. Responses to the various tests were graded from 0 (no response) to 5 (maximal or optimal response). ${ }^{18} \mathrm{~A}$ total of 10 rats were successfully established as HIBD models.

\section{Learning and Spatial Memory in HIBD Rats, Determined by Morris Water Maze}

Morris water maze experiments were conducted 3 days after HIBD surgery. A $100-\mathrm{cm}$-diameter round pool was divided into separate regions (I, II, III, and IV regions). A hidden, 6$\mathrm{cm}$-diameter platform was placed $1 \mathrm{~cm}$ below the water surface at region IV. Pool water $\left(25^{\circ} \mathrm{C}\right)$ was made opaque by adding an appropriate amount of milk, with a camera placed right above the center of the pool. Rat swimming was traced using an automatic video recording system. The rats were trained once a day for 5 days. The rats were placed in pool water with their head facing the wall of the pool. The time spent in finding the hidden platform was recorded. Rats that failed to find the platform within 90 seconds were guided to the platform and kept for 15 seconds. In this case, the time spent was recorded as 90 seconds. Probe test was performed at day 6 of the experiment. The hidden platform was then removed. The rats were placed in pool water at the entry point in the order of I-II-III-IV. Time spent was 
recorded when rats were in region IV, where the hidden platform was originally placed. After the Morris water maze test, four HIBD rats were euthanized by i.p. injection with $0.3 \%$ pentobarbital sodium solution $(30 \mathrm{mg} / \mathrm{kg})$. The hippocampus of the euthanized rats was harvested and stored at $-80^{\circ} \mathrm{C}$ for subsequent experiments. ${ }^{19}$

\section{Establishment of Rat H19 Depletion or Overexpression Models}

A total of 30 HIBD rats were prepared. The rats were then injected with adenovirus (Genomeditech, Shanghai, China) expressing H19 $(n=10)$, adenovirus vectors carrying siRNA against H19 $(n=10)$, or blank adenovirus vectors $(n=10)$.

\section{Culture of Primary Neuron Cells}

Male Sprague-Dawley rats were subjected to carbon dioxide asphyxiation $(n=6)$ within 24 hours of birth, and the bilateral cerebral cortex was collected. The meninges, cerebellum, hippocampus, and brain medulla were removed, and the brain tissues were cut and trypsinized at $37^{\circ} \mathrm{C}$ for 15 minutes. Cell suspension was separated by differential adhesion method for 10 minutes. The unattached cells were cultured in a $75-\mathrm{cm}^{2}$ culture bottle coated with L-polylysine in $5 \% \mathrm{CO}_{2}$ at $37^{\circ} \mathrm{C}$. Half of the culture medium was renewed every 3 days. At $85 \%$ to $95 \%$ confluency, the cells were oscillated at $37^{\circ} \mathrm{C}(150 \times g ; 5$ hours $)$ to remove the cell supernatant.

\section{OGD Model in Rat Primary Neuron Cells}

To simulate ischemic and hypoxic conditions in vitro, the attached neurons from the rats were trypsinized and subcultured as an oxygen-glucose deprivation (OGD) cell model. Dulbecco's modified Eagle's medium was replaced by Hanks' balanced salt solution without glucose. The neurons were incubated in a hypoxic chamber (mic-101; Billups-Rothenberg, Del Mar, CA; 95\% nitrogen and 5\% $\mathrm{CO}_{2}$ ) for 60 minutes. The cells were then incubated under a normoxic/normoglycemic condition for 6 hours. $^{20,21}$

\section{Cell Grouping and Transfection}

$80 \%$ confluent primary neuronal cells were infected with adenovirus vectors (Genomeditech) carrying H19 overexpression plasmids, miR-107 mimics, or their negative control (NC) plasmids. After infection, the cells were incubated at $37^{\circ} \mathrm{C}, 5 \% \mathrm{CO}_{2}$, and saturated humidity. After 6 hours, the medium was renewed with Dulbecco's modified Eagle's medium (Santa Cruz Biotechnology, Inc., Dallas, TX) containing $10 \%$ fetal bovine serum for 72 hours of further incubation. ${ }^{22}$ The transfection reagents were purchased from Shanghai GenePharma Co Ltd (Shanghai, China). The experiment was repeated three times.

\section{Cell RNA Isolation and Quantitation for RT-qPCR}

Total cell RNA was extracted by TRIZOL (Beijing Solarbio Science \& Technology Co., Ltd., Beijing, China), and the RNA concentration was determined. The primer sequences are listed in Table 2 (TaKaRa Biotechnology Co, Ltd, Liaoning, China). cDNA was synthesized using a cDNA reverse transcription kit (D0401; Harbin HaiGene Co, Ltd, Harbin, China). Reverse transcription of antisense miRNA was conducted using a one-step miRNA reverse transcription kit (D1801; Harbin HaiGene Co, Ltd). Next, cDNA (1 $\mu \mathrm{L})$ was employed for quantitative fluorescence, and quantitative PCR of coding genes (AQ101-02; Beijing TransGen Biotech Co, Ltd, Beijing, China) was conducted using a quantitative RT-PCR (RT-qPCR) system (ABI ViiA7; Da An Gene Co, Ltd, Guangzhou, China). Glyceraldehyde-3-phosphate dehydrogenase $(G A P D H)$ and $U 6$ were used as internal references. The $2^{-\Delta \Delta \mathrm{Ct}}$ method was applied to quantify the expressions of target genes. ${ }^{23}$ The experiment was repeated three times.

\section{Protein Extraction and Western Blot Analysis}

Western blot analysis was conducted as previously reported. ${ }^{24,25}$ Total protein was extracted from the tissues or neurons using radioimmunoprecipitation assay lysis (R0010; Beijing Solarbio Science \& Technology Co., Ltd.) at $4^{\circ} \mathrm{C}$ for 15 minutes, and centrifuged at $24,000 \times g$ for 15 minutes. The protein concentration of each sample was determined using a bicinchoninic acid kit (20201ES76; YeSen Biotechnology Co Ltd, Shanghai, China). Equal amounts of proteins at approximately $15 \mu \mathrm{g}$ were fractionated via $8 \%$ to $14 \%$ SDS-PAGE gel for 1 hour. The separated proteins were then transferred onto a polyvinylidene difluoride membrane. The membrane was blocked in 5\% nonfat milk solution. The proteins were incubated with primary antibodies VEGF (ab32152; 1:1000; Abcam, Cambridge, UK), cleaved caspase-3 (ab13847; 1:500; Abcam), caspase-3 (ab32499; 1:10,000; Abcam), B-cell

Table 2 Primers for Quantitative RT-PCR

\begin{tabular}{ll}
\hline Gene & Primer $\left(5^{\prime}-3^{\prime}\right)$ \\
\hline LncRNA H19 & F: 5'-CAAGATGGGGGGGTCAGAC-3' \\
RiR-107 & 5'-GAGCCACTTCTGAACCTGCT-3' \\
& F: 5'-ATACCGCTCGAGTGCCATGTGT \\
& CCACTGAAT-3' \\
& R: $5^{\prime}$-ATACCGCTCGAGTTCCATGCCT \\
CAACTCC-3' \\
VEGF & F: 5'-TGTGCGGGCTGCTGCAATGAT-3' \\
GAPDH & R: 5'-TGTGCTGGCTTTGGTGAGGTTTGA-3' \\
& F: 5'-GATGGTGAAGGTCGGTGTGA-3' \\
U6 & R: 5'-TGAACTTGCCGTGGGTAGAG-3' \\
& F: 5'-GCCGCATACAGAGAAGATTA-3' \\
& R: 5'-AGTGCAGGGTCCGAGGTA-3' \\
\hline
\end{tabular}

GAPDH, glyceraldehyde-3-phosphate dehydrogenase; F, forward; LncRNA H19, long noncoding RNA H19; R, reverse; VEGF, vascular endothelial growth factor. 
lymphoma-2 (Bcl-2; ab196495; 1:1000; Abcam), Bcl2-associated X (Bax; ab232479; 1:1000; Abcam), and GAPDH (ab8245; 1:1000; Abcam) overnight at $4^{\circ} \mathrm{C}$. After washing with Tris-buffered saline solution containing Tween 20 three times (5 minutes each), the membrane was incubated with horseradish peroxidase-labeled goat anti-rabbit $\mathrm{IgG}$ (ab205718; 1:20,000; Abcam) or goat anti-rat (ab6789; 1:5000; Abcam) at room temperature for 1 hour. The membrane was then washed three times with Tris-buffered saline solution containing Tween 20 (5 minutes each) and subsequently developed. Data were semiquantitatively analyzed using ImageJ software version 1.8.0 (NIH, Bethesda, MD; http:// imagej.nih.gov/ij). The relative expression of the target protein was determined on the basis of the ratio of the gray value between the target protein and GAPDH.

\section{Target Gene Analysis by Dual-Luciferase Reporter Gene Assay}

Target gene analysis of H19 and miR-107 and of miR-107 and $V E G F$ was each performed using a web-based biological prediction resource. In addition, the predicted miR-107 binding sites on the $3^{\prime}$-UTR in $\mathrm{H} 19$ and $V E G F$ were further confirmed using dual-luciferase reporter gene essay. Dual-luciferase reporter gene vectors such as wild-type (WT) miR-107, WT $V E G F$, and their mutants (MUTs) with $\mathrm{H} 19$ binding sites were subsequently constructed. The two reporter vectors were then cotransfected with miR-107 mimics into the HEK293T cells. After transfection for 24 hours, the cells were digested using TransDetect Double-Luciferase Reporter Assay Kit (FR20101; Beijing TransGen Biotech Co, Ltd, Beijing, China), and the supernatant was then collected. Luciferase activity was detected using a Dual-Luciferase Reporter Assay System kit (E1910; Promega Corp., Madison, WI). Luciferase reaction reagent $(100 \mu \mathrm{L})$ was added to the cell lysate $(20 \mu \mathrm{L})$ at room temperature to measure the Firefly luciferase activity. Luciferase reaction reagent II $(100 \mu \mathrm{L})$ was then added to determine the Renilla luciferase activity. The luciferase activity was expressed as the ratio between Firefly/Renilla luciferase. The experiment was repeated three times.

\section{Immunofluorescence Staining}

Immunofluorescence staining was conducted using a previously reported method. ${ }^{10,26}$ Briefly, the cells were fixed using $4 \%$ paraformaldehyde for 30 minutes, and then blocked in $3 \%$ bovine serum albumin for 30 minutes. Antibodies against $\mathrm{Bcl}-2$ (ab32370; 1:1000; Abcam), Bax (ab32503; 1:1000; Abcam), microtubule-associated protein 2 (ab5392; 1:10,000; Abcam), and P- $\tau$ (EPR2731; 1:50; Abcam) were added, respectively, and incubated overnight at $4{ }^{\circ} \mathrm{C}$. The cells were then incubated with green and red fluorescent antibodies (Interwiki, Baltimore, $\mathrm{MD})$ at room temperature for 2 hours. Thereafter, the cells were fixed, and imaged under a fluorescence microscope (Olympus, Tokyo, Japan) after staining with DAPI nuclear-specific markers for 5 minutes.

\section{RNA Fluorescence in Situ Hybridization}

H19 sequence and the specific probe of miR-107 were subjected to fluorescent hybridization. In brief, a cy5labeled probe was used for H19, whereas a fluorescent dye-labeled probe was used for miR-107. The nucleus was stained with DAPI. All procedures were performed in accordance with the manufacturer's instructions (Gene Pharma, Shanghai, China). All images were captured using a Zeiss LSM880 NLO $(2+1$ with BIG) confocal microscope system (Leica Microsystems, Mannheim, Germany).

\section{RNA Immunoprecipitation}

RNA immunoprecipitation was conducted using an RNA immunoprecipitation kit (Millipore, Bedford, MA). The cell supernatant was incubated with treated beads for 6 hours and washed with RNA immunoprecipitation rinsing buffer six times. Purified RNA was used for RT-qPCR. ${ }^{27}$

\section{RNA Pull Down}

WT-bio-LncRNA H19 and MUT-bio-LncRNA H19 (50 nmol/ L; biotin labeled) were transfected into 293T cells. After 48 hours, the cells were collected and washed with phosphatebuffered saline, followed by incubation in specific lysis buffer (Ambion, Austin, TX) for 10 minutes, and subsequently centrifuged at $33,000 \times g$ for 10 minutes to obtain the supernatant. Protein lysate was incubated with M-280 streptavidin magnetic beads (S3762; Sigma-Aldrich, St. Louis, MO) precoated with RNase-free bovine serum albumin and yeast tRNA (TRNABAK-RO; Sigma-Aldrich). Magnetic beads were incubated at $4{ }^{\circ} \mathrm{C}$ for 3 hours and washed twice with precooled lysis buffer, three times with low-salt buffer, and once with high-salt buffer. TRIzol was used to purify the bound RNA, and the expression of $\mathrm{H} 19$ was determined by RT-qPCR. ${ }^{28}$

\section{Terminal Deoxynucleotidyl Transferase-Mediated dUTP Nick-End Labeling Staining}

The brains, harvested after euthanizing the rats, were rinsed under running water, dehydrated in gradient alcohol, cleared in xylene, and embedded. Paraffin-embedded brain tissues were sliced into sections ( $5 \mu \mathrm{m}$ thick) and dried overnight. After dewaxing, the sections were stained with $0.5 \%$ hematoxylin, rinsed with water, differentiated with $75 \%$ hydrochloric acid alcohol, and washed with water. After treatment with ammonia bluing solution, the samples were stained with eosin, dehydrated in gradient ethanol, cleared in xylene, mounted in neutral resin, and dried overnight. Next, the sections were dewaxed and incubated with protease $\mathrm{K}$ working solution (protease $\mathrm{K}$ storage solution diluted by phosphatebuffered saline at a ratio of $1: 9$ ) at $37^{\circ} \mathrm{C}$ for 30 minutes. The samples were then washed three times with phosphatebuffered saline (5 minutes each), followed by rupture of cell membranes (consistent with immunofluorescence staining). 

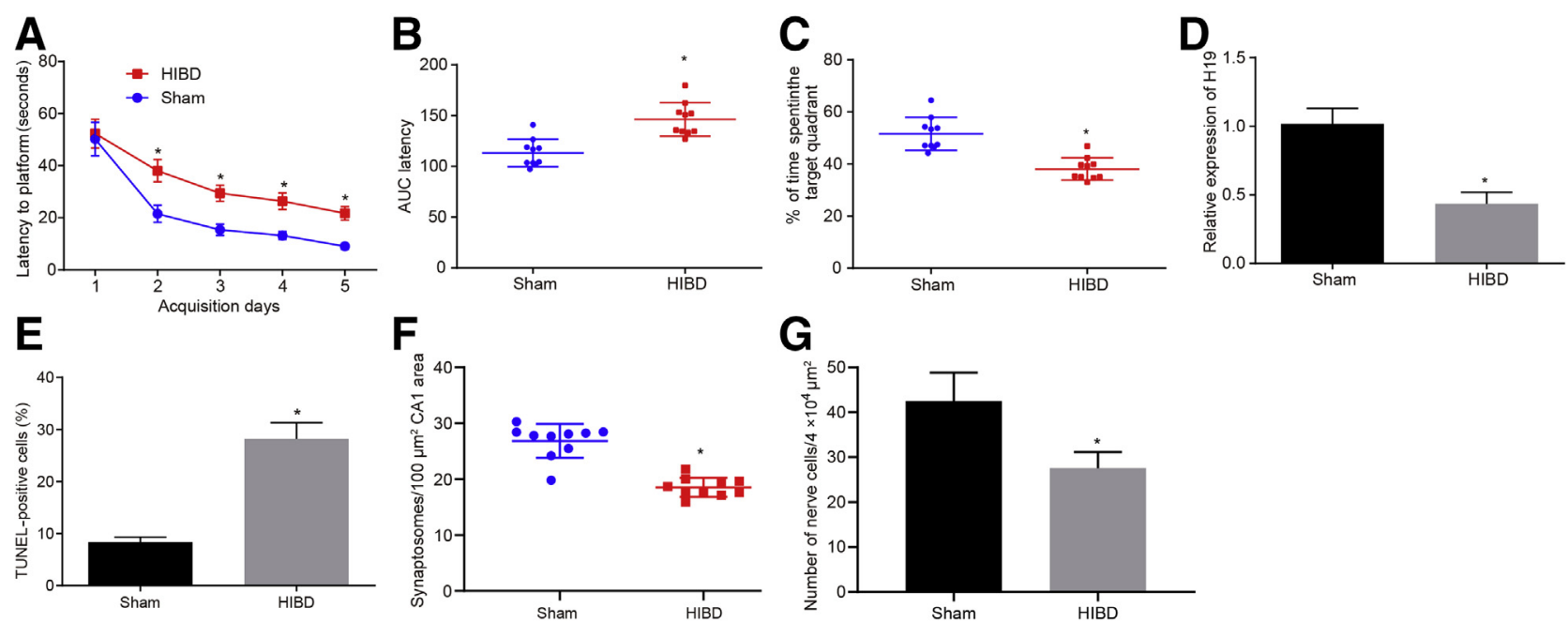

\section{G}
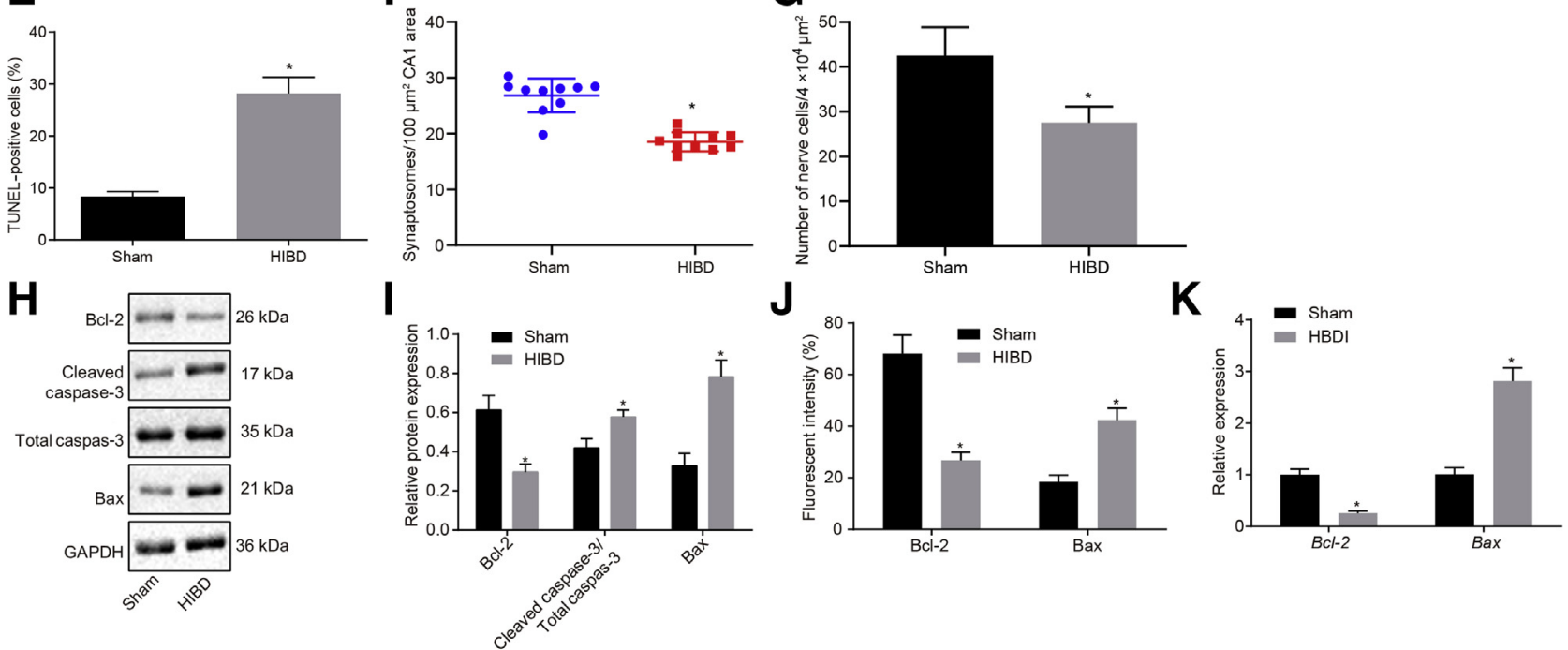

Figure 1 Molecular and behavioral characteristics in hypoxic-ischemic brain damage (HIBD) rats. A: Time spent to find the hidden platform by HIBD rats during Morris water maze test. B: Travel and comprehensive latency times in HIBD rats. C: Time spent in the target region by HIBD rats when the hidden platform was removed. D: Long noncoding RNA H19 (H19) mRNA expression level, as determined by quantitative RT-PCR. E: Neuronal apoptosis, detected by terminal deoxynucleotidyl transferase-mediated dUTP nick-end labeling (TUNEL) staining. F: Synaptic density in hippocampus regions from HIBD rats. G: Number of neurons in HIBD rats, visualized by Nissl staining. H: Representative images of B-cell lymphoma-2 (Bcl-2), Bcl-2-associated X (Bax), total caspase3, and cleaved caspase-3 protein bands. I: Protein levels of Bcl-2, Bax, total caspase-3, and cleaved caspase-3 by Western blot analysis. J: Fluorescence intensity of Bcl-2 and Bax proteins by immunofluorescence staining. K: mRNA levels of Bcl-2 and Bax. Comparisons of data between two groups were performed using independent-sample $t$-test, and comparisons of data between three groups were performed using repeated measurement analysis of variance, followed by the Tukey post hoc test. Measurement data are expressed as means \pm SD (A-G and $\mathbf{I}-\mathbf{K}) .{ }^{*} P<0.05$ versus sham-operated rats. AUC, area under the curve; GAPDH, glyceraldehyde-3-phosphate dehydrogenase.

Reagent 1 [terminal deoxynucleotidyl transferase (TdT)] and reagent 2 (dUTP) were mixed (2:29) in terminal deoxynucleotidyl transferase-mediated dUTP nick-end labeling reagent kit (11684817910; Roche Diagnostics GmbH, Mannheim, Germany) and added to the samples. The sections were subsequently incubated in a wet box at $37^{\circ} \mathrm{C}$ for 2 hours. Randomly selected fields in each section were imaged using a confocal microscope. Apoptosis index was calculated as the ratio of positive values total.

\section{Observation of Hippocampal Neurons after Nissl Staining}

Paraffin-embedded hippocampus sections were dewaxed at $37^{\circ} \mathrm{C}$ and stained with cresol violet for 45 minutes. Next, the samples were rinsed and differentiated using Nissl differentiation solution under a microscope. The sections were then immersed in distilled water to terminate staining. The sections were dehydrated by ethanol and mounted in gum.
Morphologic changes of hippocampal neurons were observed under an optical microscope. ${ }^{29}$

\section{Observation of Synapses by Electron Microscope}

After deep anesthesia, the rats were perfused with phosphate-buffered saline supplemented with $2 \%$ glutaraldehyde and 3\% paraformaldehyde subcutaneously. The brain sections were placed in cold $1 \% \mathrm{OsO}_{4}$ osmium oxide for 1 hour. Ultrathin sections $(90 \mathrm{~nm})$ were stained with uranyl acetate and lead acetate. Synaptic vesicles and post-tactile synapse density were observed by investigators blinded to the experimental groups under a JEOL 200CX electron microscope (100 $\mathrm{kV}$; JEOL, Tokyo, Japan). ${ }^{30}$

\section{Statistical Analysis}

Statistical analyses were performed using SPSS 21.0 software (IBM Corp., Armonk, NY). Data were expressed as 
A P- $\tau$

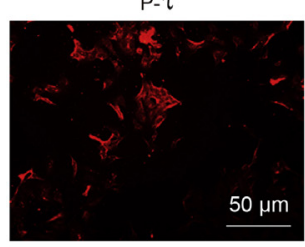

C

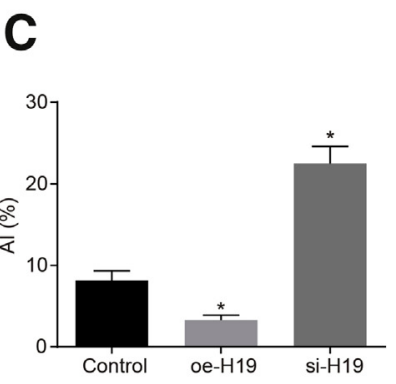

DAP

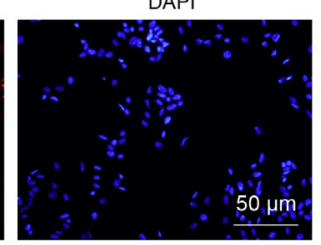

D Control

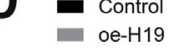

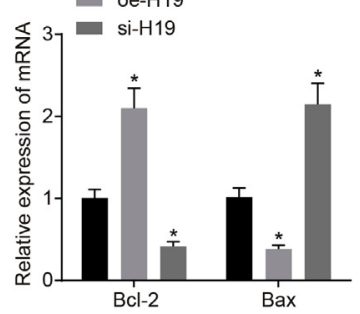

MAP2
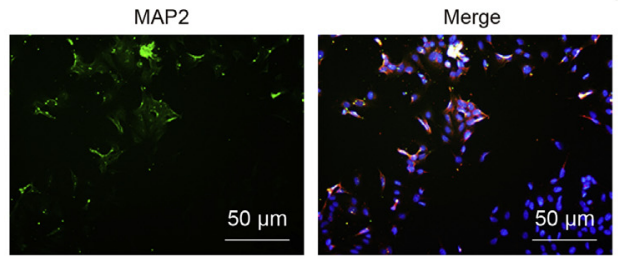

E

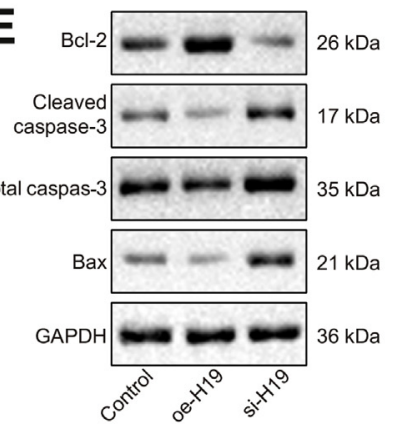

B
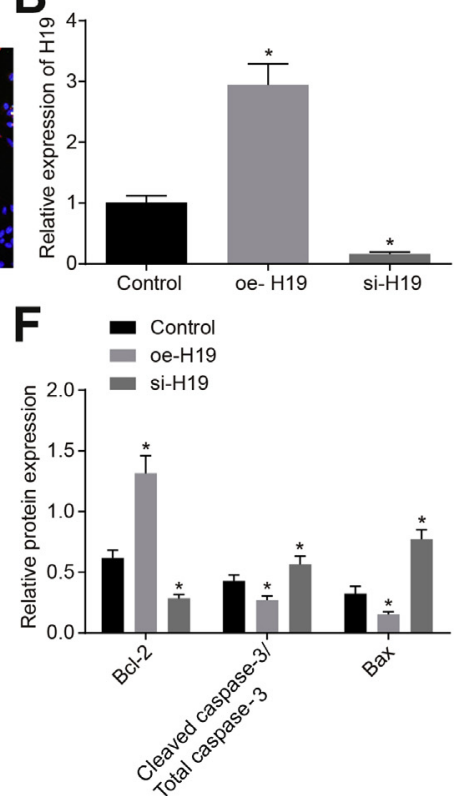

Figure 2 Long noncoding RNA H19 (H19) overexpression inhibits neuronal apoptosis in hypoxic-ischemic brain damage rats. A: Representative micrographs showing expression levels of neuronal protein markers microtubule-associated protein 2 (MAP2) and P- $\tau$ via immunofluorescence staining. B: H19 expression determined with quantitative RT-PCR (RT-qPCR) in cells infected with adenovirus vectors carrying siRNA against $\mathrm{H} 19$ (si-H19) or cells infected with adenovirus vectors carrying $\mathrm{H} 19$ overexpression (oe-H19). C: The apoptotic index (AI) of neurons, determined by terminal deoxynucleotidyl transferasemediated dUTP nick-end labeling staining. D: mRNA levels of B-cell lymphoma-2 (Bcl-2) and Bcl-2-associated X (Bax), determined by RT-qPCR. B and D: Comparisons of data were performed using one-way analysis of variance, followed by the Tukey post hoc test; the experiment was repeated three times. E: Representative images of Bcl-2, Bax, total caspase-3, and cleaved caspase-3 protein bands. F: Protein levels of Bcl-2, Bax, total caspase-3, and cleaved caspase-3, determined by Western blot analysis. Measurement data are expressed as means \pm SD (B-D and $\mathbf{F}) . n=4$ (B-D and $\mathbf{F})$. ${ }^{*} P<0.05$ versus control. Scale bars $=50 \mu \mathrm{m}(\mathbf{A})$. Control, cells without treatment; GAPDH, glyceraldehyde-3-phosphate dehydrogenase.

means \pm SD. All data sets were evaluated for normal distribution and homogeneity of variance. Comparison of data from two groups was performed using unpaired $t$-test. Comparisons between multiple groups were made by one-way analysis of variance, followed by the post hoc Tukey test. Comparisons between multiple groups at different time points were conducted by repeated-measures analysis of variance, followed by the post hoc Tukey test.

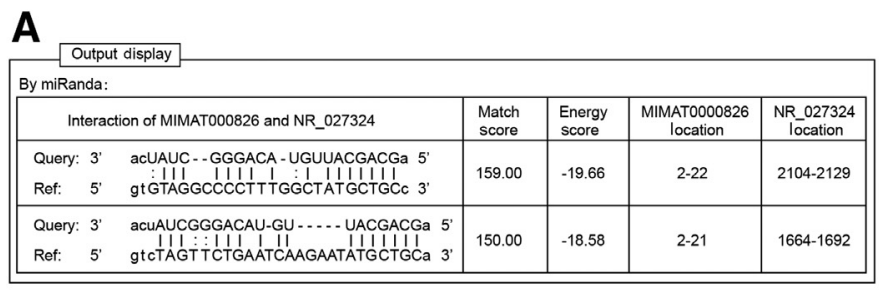

C

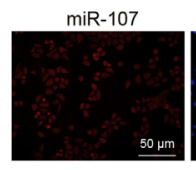
DAPI

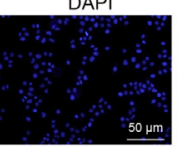

$\mathrm{H} 19$

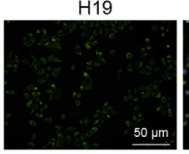

\section{D}

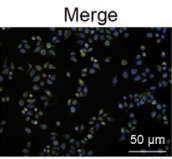

B

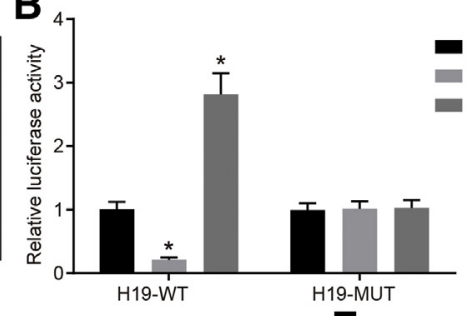

E

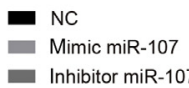

Inhibitor miR-107

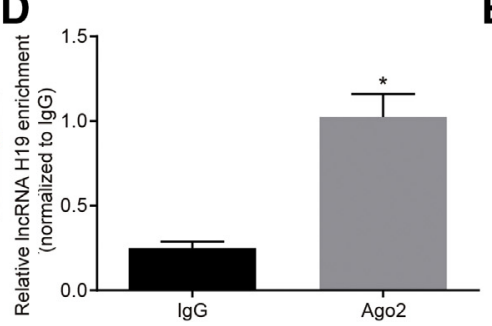

- NC-bio-probe - WT-bio-miR-107 - MUT-bio-miR-107

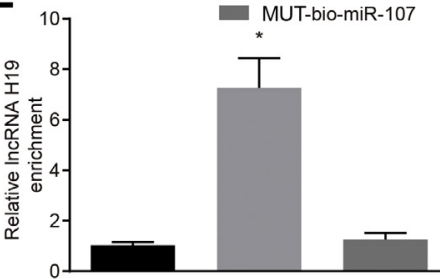

Figure 3 Binding relationship between long noncoding RNA H19 (H19) and miR-107. A: Binding relationship between H19 and miR-107, as predicted by a web-based resource. B: Binding relationship between H19 and miR-107, determined by dual-luciferase reporter gene essay. C: Representative micrographs showing colocalization of $\mathrm{H} 19$ and miR-107 in cytoplasm, detected by fluorescence in situ hybridization. D: Expression of H19 after binding to argonaute 2 (Ago2), determined by RNA immunoprecipitation. D: Data comparisons using independent-sample $t$-test; the experiment was repeated three times. E: Relationship between H19 and miR-107, determined by RNA pull-down assay. B and E: Data comparisons using one-way analysis of variance, followed by the Tukey post hoc test. Measurement data are depicted as means $\pm \mathrm{SD}(\mathbf{B}, \mathbf{D}$, and $\mathbf{E})$. ${ }^{*} P<0.05$ versus cells treated with negative control (NC), Ago2, or NC-bio probe. Scale bars $=50 \mu \mathrm{m}(\mathbf{C})$. IncRNA, long noncoding RNA; MUT, mutant; Ref, reference; WT, wild type. 
Differences were considered to be of statistical significance when $P<0.05$.

\section{Results}

\section{Establishment of HIBD Rat Model}

The cognitive functions of the HIBD rats were first determined. In Morris water maze test, HIBD rats spent less time in the target region when the hidden platform was removed (Figure 1, A-C), suggestive of memory deficits in the rats. H19 expression was determined using RT-qPCR to verify the role of H19 in HIBD. H19 mRNA expression was reduced in the neurons of the HIBD rats when compared with that in sham-operated rats $(P<0.05)$ (Figure 1D). The terminal deoxynucleotidyl transferase-mediated dUTP nick-end labeling assay to detect apoptosis of the HIBD neurons indicated an increase in the apoptosis of neurons in HIBD rats compared with that in sham-operated rats $(P<0.05)$ (Figure $1 \mathrm{E})$. Relative to the sham-operated rats, a decrease in the density of synaptosome in the hippocampus was observed in HIBD rats (Figure 1F). Moreover, the number of neurons in HIBD rats was lower in CA1 regions of hippocampus when compared with that in sham-operated rats $(P<0.05)$ (Figure $1 \mathrm{G})$. Immunofluorescence staining revealed decreased $\mathrm{Bcl}-2$ protein level and increased Bax protein level in HIBD in comparison to that in the shamoperated rats $(P<0.05)$ (Figure $1 \mathrm{~J})$. Moreover, Bcl-2 mRNA (Figure $1 \mathrm{~K}$ ) and protein expression (Figure 1, H and I) were each reduced in HIBD when compared with that in sham-operated rats. In contrast, Bax, total and cleaved caspase-3 mRNA, and protein expression were all elevated $(P<0.05)$. The aforementioned results indicate that increased HIBD is related to increased apoptosis of neurons in HIBD rats.

Altogether, these data suggest hippocampal injury and impaired cognitive function in HIBD rat models.

\section{H19 Overexpression Inhibits Neuronal Apoptosis in HIBD Rats}

Neurons were isolated from HIBD rats, and the expression levels of neuronal protein markers (microtubule-associated protein 2 and P- $\tau$ ) were determined. ${ }^{31,32}$ Immunofluorescence staining revealed the presence of microtubuleassociated protein 2 and P- $\tau$, confirming the successful
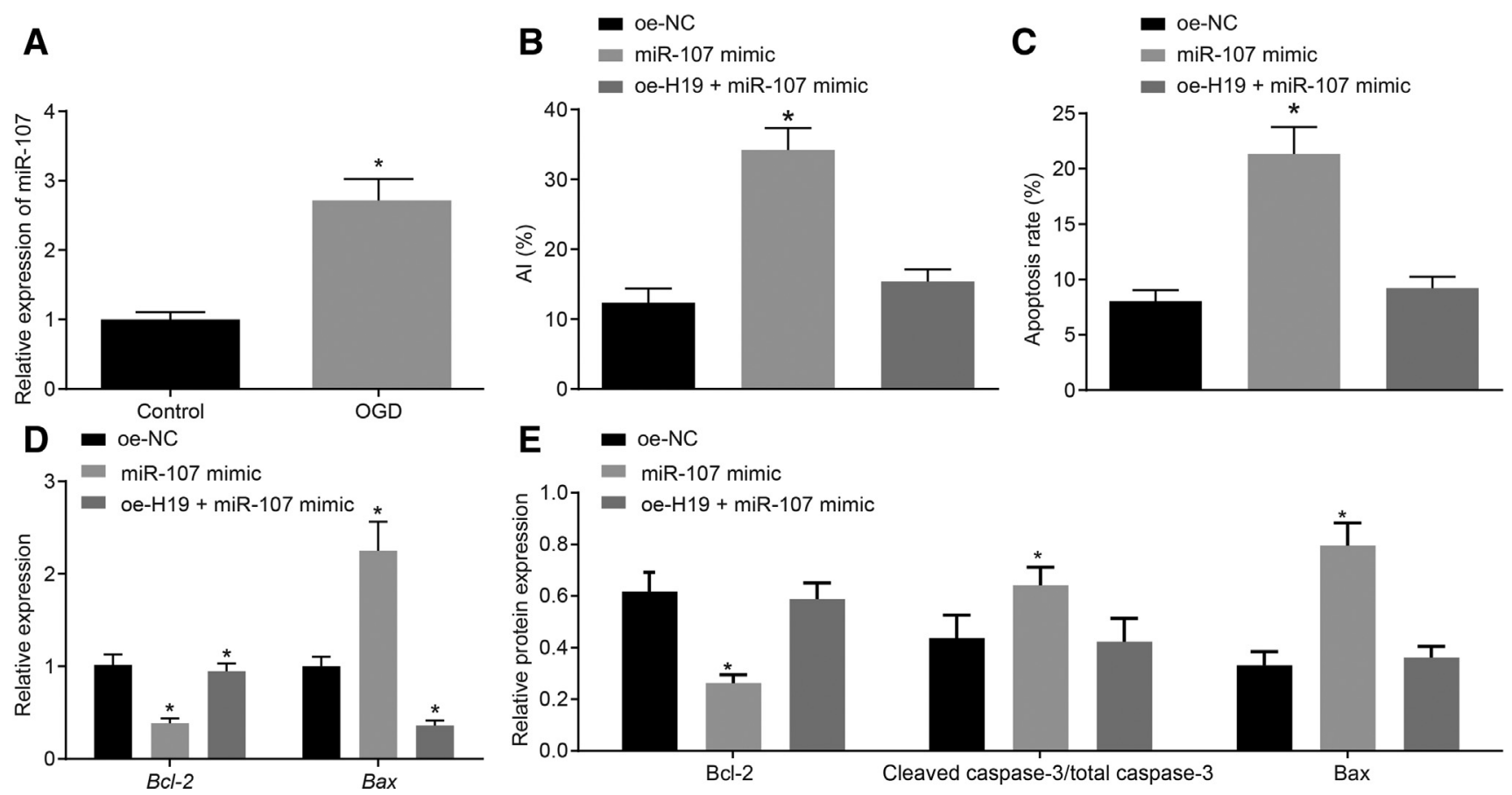

Figure 4 miR-107 up-regulation eliminates the protective effects of long noncoding RNA H19 (H19) on oxygen-glucose deprivation (OGD) neurons. A: miR-107 expression was determined in OGD cell model by quantitative RT-PCR (RT-qPCR). A: Data were compared using independent-sample $t$-test. B: The apoptotic index (AI) of neurons, determined by terminal deoxynucleotidyl transferase-mediated dUTP nick-end labeling staining. C: Neuron apoptosis, detected by flow cytometry. D: mRNA levels of B-cell lymphoma-2 (Bcl-2) and Bcl-2-associated X (Bax), as determined by RT-qPCR. E: Protein levels of Bcl-2, Bax, total caspase-3, and cleaved caspase-3, as determined by Western blot analysis. D and E: Data were compared using one-way analysis of variance, followed by the Tukey post hoc test; the experiment was repeated three times. Measurement data are depicted as means \pm SD $(\mathbf{A}-\mathbf{E})$. ${ }^{*} P<0.05$ versus cells without infection or cell injected with adenovirus vectors carrying negative control for miR-107 mimics (oe-NC). miR-107 mimic, cell injected with adenovirus vectors carrying miR-107 mimics; oe-H19 + miR-107 mimic, cell injected with adenovirus vectors carrying H19 overexpression plasmids and carrying miR-107 mimics. 
A

\begin{tabular}{|c|c|c|c|c|c|}
\hline miR name & Transcript name & $\begin{array}{c}\text { Leftmost position of } \\
\text { predicted target site }\end{array}$ & $\begin{array}{c}\text { Folding energy } \\
\text { (in -Kcal/mol) }\end{array}$ & Heteroduplex & Pvalue \\
\hline rno-miR-107 & VEGF & 5203 & -14.00 & $\begin{array}{c}\text { CCTCGGTCACATCTCTCTGCTGCT } \\
:|:||||||||| \mid\end{array}$ & 0.0731 \\
\hline
\end{tabular}
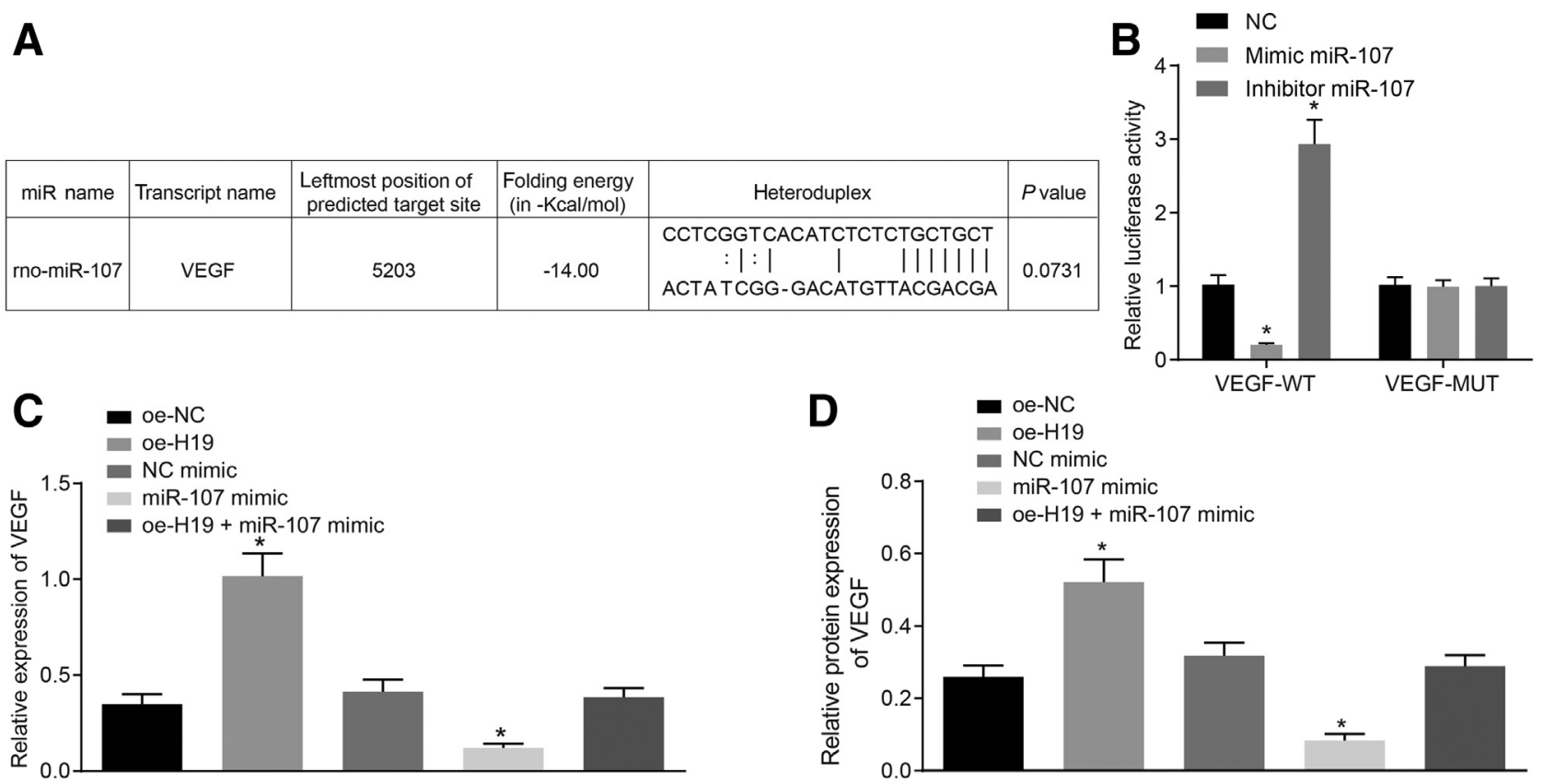

Figure 5 Binding relationship between vascular endothelial growth factor (VEGF) and miR-107. A: Diagram showing binding sites between miR-107 and VEGF, determined by bioinformatic analysis. B: Binding relationship between miR-107 and VEGF, determined by dual-luciferase reporter gene essay. C: VEGF mRNA level in different groups, quantified by quantitative RT-PCR. D: VEGF protein level, evaluated by Western blot analysis. B-D: Data were compared using one-way analysis of variance (Tukey post hoc test); the experiment was repeated three times. Measurement data are depicted as means \pm SD $(\mathbf{B}-\mathbf{D}) .{ }^{*} P<0.05$ versus cell injected with adenovirus vectors carrying negative control for miR-107 mimics (oe-NC). MUT, mutant; NC, negative control; oe-H19, cell injected with adenovirus vectors carrying long noncoding RNA H19 overexpression plasmids; WT, wild type.

isolation of neurons (Figure 2A). The isolated neurons were then infected with adenovirus vectors carrying either siRNA against H19 or H19 overexpression. H19 expression was increased on infection with $\mathrm{H} 19$ overexpression $(P<0.05)$ and decreased on infection of vector with siRNA against H19 $(P<0.05)$ (Figure 2B), confirming that the infection was successful. H19 overexpression reduced, whereas H19 depletion promoted, apoptosis $(P<0.05)$ (Figure $2 \mathrm{C})$. Bcl2 mRNA and protein expression were each reduced by $\mathrm{H} 19$ overexpression, whereas $\mathrm{H} 19$ depletion resulted in the increase in Bcl-2 expression $(P<0.05)$ (Figure $2 \mathrm{D}$ ). In contrast, the mRNA and protein levels of Bax and total cleaved caspase- 3 were all diminished in the presence of H19 overexpression, whereas H19 depletion increased the aforementioned expression levels $(P<0.05)$ (Figure 2, E and F). The above-mentioned data implicated the suppression of $\mathrm{H} 19$ on neuron apoptosis.

\section{Binding Relation between $\mathrm{H} 19$ and miR-107}

The activation of $\mathrm{H} 19$ is reported to suppress miR-107 expression and thus promote cell cycle progression of non-small-cell lung cancer. ${ }^{13}$ The bioinformatic web-based resource Targetscan (http://www.targetscan.org/vert_71, last accessed December 14, 2018), adopted to validate targeting relationships in HIBD, identified a specific binding site between the $3^{\prime}$-UTR and miR-107 sequence, suggesting that H19 can target miR-107 (Figure 3A). A dual-luciferase report gene assay was employed to identify the binding relationship between H19 and miR-107. Treatment with miR-107 mimic cotransfected with H19 WT led to an increase in $\mathrm{H} 19$ expression, whereas $\mathrm{H} 19$ expression was decreased after treatment with miR-107 inhibitor (Figure 3B). However, these changes were not observed in response to H19 MUT (Figure 3B). Colocalization of H19 and miR-107 in cytoplasm was demonstrated by fluorescence in situ hybridization (Figure 3C). Furthermore, H19 expression increased when bound to argonaute 2 (Ago2) versus $\operatorname{IgG}(P<0.05)$ (Figure 3D). H19 expression increased in HEK 293 cells transfected with WT-miR-107 when compared with those with MUT-miR-107 $(P<0.05)$ (Figure 3E). These results suggested that $\mathrm{H} 19$ competitively bound to miR-107. miR-107 plays a vital role in the pathogenesis of traumatic brain injury and neurodegenerative diseases, such as Alzheimer disease. ${ }^{13}$ Therefore, H19 and miR-107 were used for further study.

\section{miR-107 Up-Regulation Eliminates the Protective Effects of H19 on OGD Neurons}

In the OGD cell model, the expression of miR-107 was increased in comparison to control $(P<0.05)$ (Figure 4A). The OGD neurons were subsequently transduced with adenovirus vectors carrying H19 overexpression plasmids, miR-107 mimics, or their negative control plasmids. OGD neurons infected with miR-107 mimic exhibited increased 

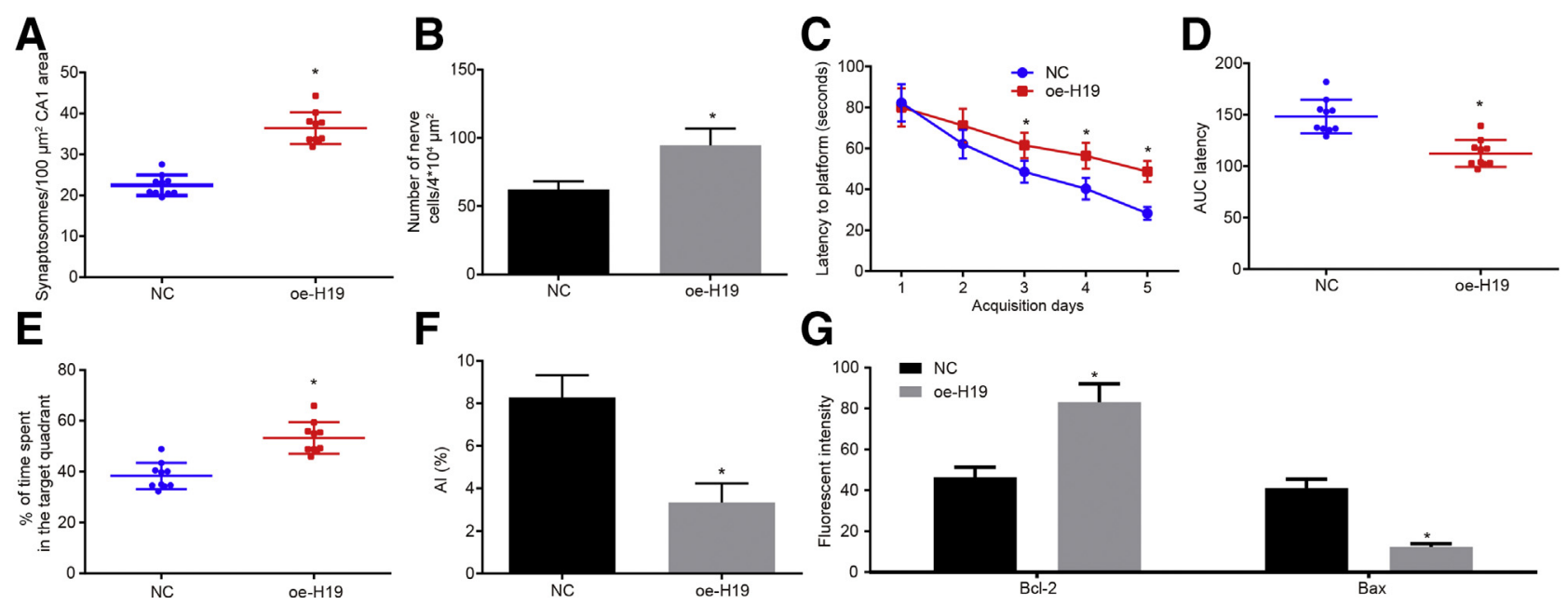

Figure 6 Long noncoding RNA H19 (H19) overexpression relieves cognitive dysfunction and inhibits neuronal apoptosis in hypoxic-ischemic brain damage (HIBD) rats. A: Synaptic density in hippocampus of HIBD rats under an electron microscope. B: The number of neurons in HIBD rats. C: Platform latency time in Morris water maze test. C: Comparison of data was performed using one-way analysis of variance, followed by the Tukey post hoc test; the experiment was repeated three times. D: Travel and comprehensive latency times in Morris water maze test. E: Time spent in the target region in HIBD rats during Morris water maze test. F: Neuronal apoptosis, detected by terminal deoxynucleotidyl transferase-mediated dUTP nick-end labeling staining. $\mathbf{D}$ and $\mathbf{F}$ : Data were compared using independent-sample $t$-test. G: Fluorescence intensity of B-cell lymphoma-2 (Bcl-2) and Bcl-2-associated X (Bax) proteins by immunofluorescence staining. Measurement data are depicted as means $\pm \mathrm{SD}(\mathbf{A}-\mathbf{G}) . n=4(\mathbf{A}-\mathbf{G}) .{ }^{*} P<0.05$ versus cells treated with negative control (NC). AI, apoptosis index; AUC, area under the curve; oe-H19, HIBD rats injected with adenovirus vectors carrying $\mathrm{H} 19$ overexpression.

neuronal apoptosis when compared with $\mathrm{NC}(P<0.05)$ (Figure 4, B-D). However, the OGD neurons infected with a combination of $\mathrm{H} 19$ overexpression plasmids and miR107 mimic displayed no significant differences (Figure 4, B-D). In addition, OGD neurons infected with miR-107 mimic displayed decreased $\mathrm{Bcl}-2 \mathrm{mRNA}$ and protein levels when compared with NC $(P<0.05)$ (Figure 4E). In contrast, total and cleaved caspase- 3 mRNA and protein levels of Bax were increased in OGD neurons infected with miR-107 mimic versus those infected with NC $(P<0.05)$ (Figure 4E). However, these changes were not observed when OGD neurons were infected with a combination of H19 overexpression plasmids and miR-107 mimic (Figure 4E). Altogether, these results suggest that increased miR-107 expression eliminates the protective effects of H19 on OGD neurons.

\section{H19 Affects VEGF Expression by Regulating miR-107}

Binding sites were identified between miR-107 and VEGF by utilizing the online bioinformatics resource (Figure 5A). Next, dual-luciferase reporter gene assay was performed for further verification of their correlation. The luciferase activity of 3'-UTR in WT-VEGF was diminished by miR-107 mimic $(P<0.05)$, whereas no significant difference was detected in the luciferase activity of $3^{\prime}$-UTR in MUT-VEGF (Figure 5B). These results suggest that miR-107 is bound to $V E G F$. Next, to further determine the role of $V E G F$, the cells were treated with $\mathrm{H} 19$ overexpression, miR-137 mimic, or a combination of the two. VEGF mRNA and protein expression levels were increased in the cells treated with overexpression $\mathrm{H} 19$ but decreased in cells treated with
miR-107 mimic when compared with treatment with $\mathrm{NC}$ $(P<0.05)$ (Figure 5, C and D). However, similar changes were not observed in cells cotransfected with overexpression H19 and miR-107 mimic (Figure 5, C and D). Taken together, these results illustrate that miR-107 binds to and decreases expression of $V E G F$.

\section{H19 Overexpression Alleviates Cognitive Dysfunction and Inhibits Neuron Apoptosis in HIBD Rats}

H19 overexpression significantly increased synaptic activity in HIBD rats $(P<0.05)$ (Figure 6A). Similarly, the number of neurons was also increased following H19 overexpression in HIBD rats $(P<0.05)$ (Figure 6B). During the Morris water maze experiment, the time taken to find the hidden platform and the time spent in the regions with a hidden platform were significantly improved following H19 overexpression in HIBD rats $(P<0.05)$ (Figure 6, C-E). These results demonstrate that H19 overexpression can alleviate cognitive dysfunction associated with HIBD.

H19 overexpression decreased neuronal apoptosis $(P<0.05)$ (Figure 6F). Immunofluorescence staining illustrated that $\mathrm{H} 19$ overexpression increased $\mathrm{Bcl}-2$ but decreased the levels of Bax protein in HIBD rats $(P<0.05)$ (Figure 6G). Taken together, the above results demonstrate that $\mathrm{H} 19$ suppresses neuronal apoptosis in HIBD rats.

\section{Discussion}

HIBD, the most common cause of brain injury in neonates, remains a major cause of diminished quality of life in 


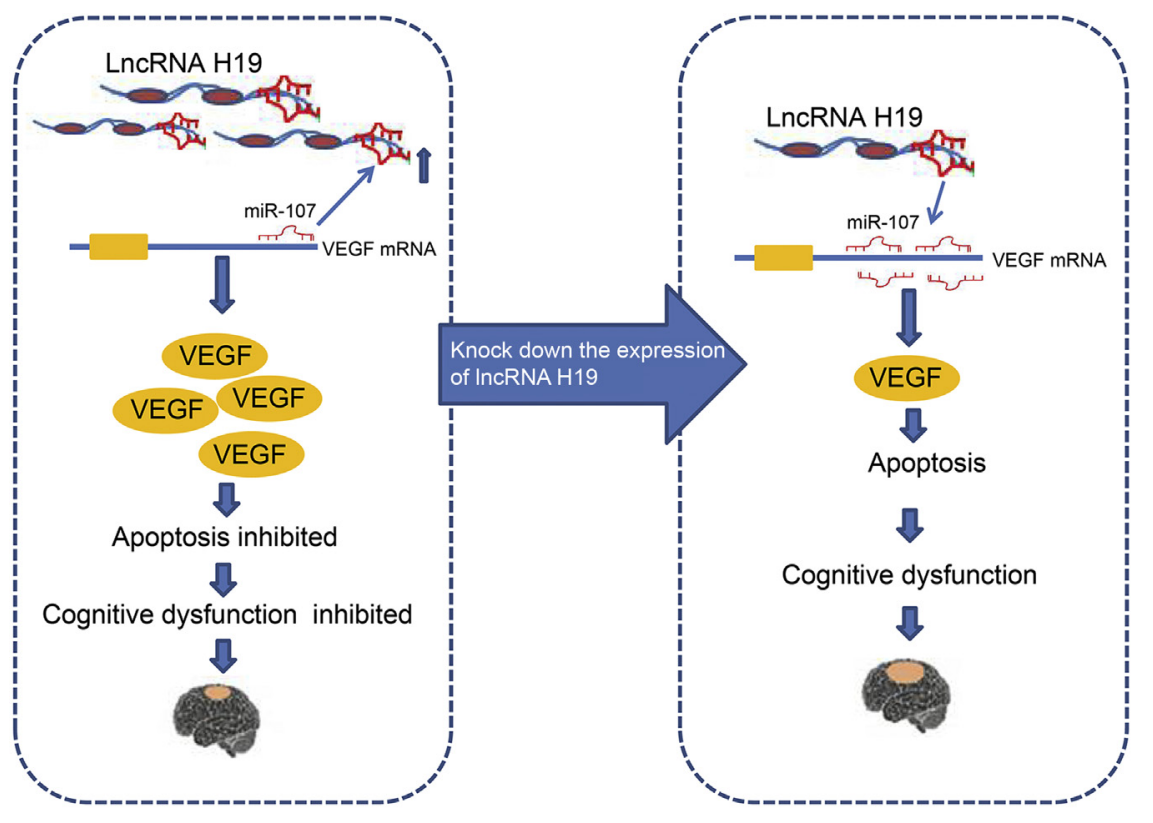

Figure 7 Schematic diagram depicting the functional role of the long noncoding RNA H19 (LncRNA H19)/miR-107/vascular endothelial growth factor (VEGF) axis in hypoxic-ischemic brain damage (HIBD). H19 expression was low in HIBD rats, which down-regulated the expression of miR-107 and up-regulated the expression of VEGF, thereby relieving cognitive dysfunction and inhibiting neuron apoptosis. LncRNA, long noncoding RNA. children around the world. ${ }^{1}$ HIBD also imposes enormous economic costs on families and emotional suffering of children; thus, effective treatment modalities are warranted. ${ }^{33}$ The current study attempted to reveal the underpinning molecular mechanisms involved in the progression of HIBD in rat and neuron cell models. The current study found that H19 expression was decreased in HIBD. More importantly, overexpression of H19 may alleviate HIBD by increasing $V E G F$ expression via the inhibition of miR-107 expression.

Initially, the current study identified that $\mathrm{H} 19$ was downregulated in neurons from HIBD rats, and that its upregulation inhibited neuronal apoptosis in HIBD rats. In a related finding, the expression of $\mathrm{H} 19$ was found repressed in hearts of mice with cardiac ischemia-reperfusion injury, whereas its elevation attenuated myocardial ischemiareperfusion injury. ${ }^{34}$ Down-regulation of H19 is also reported in infarcted myocardium, and its overexpression reportedly contributes to reduced infarct size and improved cardiac function. ${ }^{6}$ In a more recent study, elevation of $\mathrm{H} 19$ was found to correlate with alleviated OGD-induced injury. ${ }^{35}$ The current study further revealed that H19 decreased the expression of miR-107, in parallel with previous research showing that lncRNAs decrease the expression of miRNAs. ${ }^{10}$ Accumulating evidence shows that lncRNAs can bind to miRNAs and contribute to the development of HIBD. ${ }^{18}$ Multiple miRNAs exhibit upregulated expression in the adult nervous system, and several among these have been implicated in pathologic conditions, including ischemia. ${ }^{11} \mathrm{~A}$ previous study revealed dysregulation and diagnostic significance of multiple miRNAs, including miR-107, in Alzheimer disease. ${ }^{36}$ Consistent with the findings of the current study, the expression of
miR-107 has been reported to be involved in traumatic brain injury and neurodegenerative disease. ${ }^{14}$ Furthermore, the current study demonstrated that $V E G F$ is a target gene of miR-107, and that VEGF expression was increased by $\mathrm{H} 19$. $V E G F$ has been verified as a target gene of miR-126, which is negatively correlated with $V E G F$ expression. ${ }^{37}$ These results provided strong evidence that $\mathrm{H} 19$ expression is reduced in HIBD, which, in turn, leads to increased miR107 and decreased VEGF expression.

Notably, the overexpression of H19 increased the expression of Bcl-2, decreased Bax expression, reduced neuronal apoptosis, and alleviated cognitive dysfunction. Existing literature suggests that anti-apoptotic protein Bcl-2 and pro-apoptotic protein Bax enhance the secretion of cytochrome $c$, which promotes apoptosis by activating caspase- $3 .^{38} \mathrm{Bcl}-2$, Bax, and caspase-3 proteins are implicated in neuronal apoptosis, one of the main characteristics of HIBD.$^{39}$ HIBD commonly elicits hippocampal damage and subsequent hippocampal-dependent cognitive dysfunction. ${ }^{4}$ Similar to the current study, a previous study concluded that $\mathrm{H} 19$ acts as a sponge to miR-107 in the regulation of apoptosis in hippocampal neurons. ${ }^{9}$ Silencing of miR-210 has been reported to confer neuronal protection against HIBD in neonatal rats via suppression of neuronal death. ${ }^{40}$ In addition, a previous study asserted that IncRNA, along with miRNA, is of great importance in the neurodevelopment and etiological pathogenesis of HIBD. ${ }^{18}$ The up-regulation of lncRNA urothelial carcinoma associated 1 has been shown to play an inhibitory role in the apoptosis of hippocampal neurons via repression of miR-495, which ultimately relieves brain injury. ${ }^{41}$ Overall, present evidence suggests that $\mathrm{H} 19$ could be a novel potential therapeutic target for the treatment of HIBD by reducing hippocampal 
apoptosis and cognitive impairment. Moreover, the present study demonstrates that $\mathrm{H} 19$ increases the expression of $V E G F$, which is documented to possess neuroprotective properties of inducing neurogenesis and cerebral angiogenesis after brain injury. ${ }^{16} V E G F$ has also been shown to inhibit hypoxic hippocampal neuronal cell death, which suggests its significance in HIBD treatment. ${ }^{42}$

The findings of the present report must be considered in light of their limitations. As we investigated a preclinical model by simulating the disease in rats, the results should be verified in clinical studies. Because carotid ligation is a vascular accident and thus an independent stressor event apart from hypoxia, it may contribute to alerted H19 expression. Although we used a recognized model for induction of hypoxic brain injury, what proportion of the molecular sequelae may be attributed to the hypoxic component is unknown. In future studies, we intend to further refine the establishment of the animal model. However, the current findings provide preliminary evidence suggesting the clinical utility of H19 in HIBD.

\section{Conclusion}

The current study demonstrated that the expression of H19 is diminished in HIBD. Furthermore, H19 overexpression alleviated HIBD by binding to miR-107 and subsequently up-regulating $V E G F$ (Figure 7). H19 reduced neuronal apoptosis and ameliorated cognitive dysfunction through regulation of miR-107-VEGF axis in HIBD. Thus, the H19/miR-107 network has the potential to provide novel insights into treatment targets for HIBD. In the future, therapeutic strategies for HIBD may be directed toward the up-regulation of $\mathrm{H} 19$ as a potentially clinically viable approach in the prevention and treatment of HIBD. As research in this field is in the preclinical stage and a comprehensive understanding of the mechanism of action is incomplete further work is mandated. In particular, a separate analysis of male and female rats with larger sample sizes and assessment of neurobehavioral outcomes of sensorimotor function, including geotaxis reflex and cliff avoidance reflex, should be performed in the future.

\section{Acknowledgment}

We thank the reviewers for critical comments on this article.

\section{Author Contributions}

H.F., H.-F.L., and J.-P.Z. developed the conception and design; M.Y. and F.-X.Z. performed data collection; H.F. and Q.P. performed analysis and interpretation of the data; R.-R.W. and Q.-Y.W. prepared the figures; H.-F.L., Q.P., and M.Y. drafted the manuscript; all authors approved the final version of manuscript.

\section{References}

1. Yin X, Meng F, Wang Y, Wei W, Li A, Chai Y, Feng Z: Effect of hyperbaric oxygen on neurological recovery of neonatal rats following hypoxic-ischemic brain damage and its underlying mechanism. Int J Clin Exp Pathol 2013, 6:66-75

2. Wang X, Zhang J, Yang Y, Dong W, Wang F, Wang L, Li X: Progesterone attenuates cerebral edema in neonatal rats with hypoxicischemic brain damage by inhibiting the expression of matrix metalloproteinase-9 and aquaporin-4. Exp Ther Med 2013, 6: 263-267

3. Li C, Mo Z, Lei J, Li H, Fu R, Huang Y, Luo S, Zhang L: Edaravone attenuates neuronal apoptosis in hypoxic-ischemic brain damage rat model via suppression of TRAIL signaling pathway. Int J Biochem Cell Biol 2018, 99:169-177

4. Byeon JH, Kim GH, Kim JY, Sun W, Kim H, Eun BL: Cognitive dysfunction and hippocampal damage induced by hypoxic-ischemic brain injury and prolonged febrile convulsions in immature rats. J Korean Neurosurg Soc 2015, 58:22-29

5. van Velthoven CT, Kavelaars A, van Bel F, Heijnen CJ: Mesenchymal stem cell treatment after neonatal hypoxic-ischemic brain injury improves behavioral outcome and induces neuronal and oligodendrocyte regeneration. Brain Behav Immun 2010, 24: 387-393

6. Zhou M, Zou YG, Xue YZ, Wang XH, Gao H, Dong HW, Zhang Q: Long non-coding RNA H19 protects acute myocardial infarction through activating autophagy in mice. Eur Rev Med Pharmacol Sci 2018, 22:5647-5651

7. Kaur P, Liu F, Tan JR, Lim KY, Sepramaniam S, Karolina DS, Armugam A, Jeyaseelan K: Non-coding RNAs as potential neuroprotectants against ischemic brain injury. Brain Sci 2013, 3:360-395

8. Zhao F, Qu Y, Liu J, Liu H, Zhang L, Feng Y, Wang H, Gan J, Lu R, Mu D: Microarray profiling and co-expression network analysis of lncRNAs and mRNAs in neonatal rats following hypoxic-ischemic brain damage. Sci Rep 2015, 5:13850

9. Han CL, Ge M, Liu YP, Zhao XM, Wang KL, Chen N, Hu W, Zhang JG, Li L, Meng FG: Long non-coding RNA H19 contributes to apoptosis of hippocampal neurons by inhibiting let-7b in a rat model of temporal lobe epilepsy. Cell Death Dis 2018, 9:617

10. Paci P, Colombo T, Farina L: Computational analysis identifies a sponge interaction network between long non-coding RNAs and messenger RNAs in human breast cancer. BMC Syst Biol 2014, 8:83

11. Moon JM, Xu L, Giffard RG: Inhibition of microRNA-181 reduces forebrain ischemia-induced neuronal loss. J Cereb Blood Flow Metab 2013, 33:1976-1982

12. Zhou XM, Liu J, Wang Y, Zhang SL, Zhao X, Xu X, Pei J, Zhang MH: microRNA-129-5p involved in the neuroprotective effect of dexmedetomidine on hypoxic-ischemic brain injury by targeting COL3A1 through the Wnt/beta-catenin signaling pathway in neonatal rats. J Cell Biochem. 2018, 120:6908-6919

13. Cui J, Mo J, Luo M, Yu Q, Zhou S, Li T, Zhang Y, Luo W: c-Mycactivated long non-coding RNA H19 downregulates miR-107 and promotes cell cycle progression of non-small cell lung cancer. Int $\mathrm{J}$ Clin Exp Pathol 2015, 8:12400-12409

14. Wang WX, Wilfred BR, Madathil SK, Tang G, Hu Y, Dimayuga J, Stromberg AJ, Huang Q, Saatman KE, Nelson PT: miR-107 regulates granulin/progranulin with implications for traumatic brain injury and neurodegenerative disease. Am J Pathol 2010, 177:334-345

15. Thau-Zuchman O, Shohami E, Alexandrovich AG, Leker RR: Vascular endothelial growth factor increases neurogenesis after traumatic brain injury. J Cereb Blood Flow Metab 2010, 30: $1008-1016$

16. Zhao H, Bao XJ, Wang RZ, Li GL, Gao J, Ma SH, Wei JJ, Feng M, Zhao YJ, Ma WB, Yang Y, Li YN, Kong YG: Postacute ischemia vascular endothelial growth factor transfer by transferrin-targeted 
liposomes attenuates ischemic brain injury after experimental stroke in rats. Hum Gene Ther 2011, 22:207-215

17. Committee for the Update of the Guide for the Care and Use of Laboratory Animals: National Research Council: Guide for the Care and Use of Laboratory Animals. Eighth Edition. Washington, DC, National Academies Press, 2011

18. Rice JE 3rd, Vannucci RC, Brierley JB: The influence of immaturity on hypoxic-ischemic brain damage in the rat. Ann Neurol 1981, 9: $131-141$

19. Zhou XM, Liu J, Wang Y, Zhang MH: Silencing of long noncoding RNA MEG3 enhances cerebral protection of dexmedetomidine against hypoxic-ischemic brain damage in neonatal mice by binding to miR-129-5p. J Cell Biochem 2019, 120:7978-7988

20. Guan J, Du S, Lv T, Qu S, Fu Q, Yuan Y: Oxygen-glucose deprivation preconditioning protects neurons against oxygen-glucose deprivation/reperfusion induced injury via bone morphogenetic protein-7 mediated ERK, p38 and Smad signalling pathways. Clin Exp Pharmacol Physiol 2016, 43:125-134

21. Luan P, Xu J, Ding X, Cui Q, Jiang L, Xu Y, Zhu Y, Li R, Lin G, Tian P, Zhang J: Neuroprotective effect of salvianolate on cerebral ischaemia-reperfusion injury in rats by inhibiting the caspase- 3 signal pathway. Eur J Pharmacol 2020, 872:172944

22. Ju H, Tan JY, Cao B, Song MQ, Tian ZB: Effects of miR-223 on colorectal cancer cell proliferation and apoptosis through regulating FoxO3a/BIM. Eur Rev Med Pharmacol Sci 2018, 22:3771-3778

23. Ayuk SM, Abrahamse H, Houreld NN: The role of photobiomodulation on gene expression of cell adhesion molecules in diabetic wounded fibroblasts in vitro. J Photochem Photobiol B 2016, 161:368-374

24. Pan B, Lewno MT, Wu P, Wang X: Highly dynamic changes in the activity and regulation of macroautophagy in hearts subjected to increased proteotoxic stress. Front Physiol 2019, 10:758

25. Zhang H, Pan B, Wu P, Parajuli N, Rekhter MD, Goldberg AL, Wang X: PDE1 inhibition facilitates proteasomal degradation of misfolded proteins and protects against cardiac proteinopathy. Sci Adv 2019, 5:eaaw5870

26. Pan Y, Sun L, Wang J, Fu W, Fu Y, Wang J, Tong Y, Pan B: STI571 protects neuronal cells from neurotoxic prion protein fragmentinduced apoptosis. Neuropharmacology 2015, 93:191-198

27. Guo J, Hao C, Wang C, Li L: Long noncoding RNA PVT1 modulates hepatocellular carcinoma cell proliferation and apoptosis by recruiting EZH2. Cancer Cell Int 2018, 18:98

28. Liu P, Wang H, Liang Y, Hu A, Xing R, Jiang L, Yi L, Dong J: LINC00852 promotes lung adenocarcinoma spinal metastasis by targeting S100A9. J Cancer 2018, 9:4139-4149

29. Shan Y, Sun S, Yang F, Shang N, Liu H: Dexmedetomidine protects the developing rat brain against the neurotoxicity wrought by sevoflurane: role of autophagy and Drp1-Bax signaling. Drug Des Devel Ther 2018, 12:3617-3624

30. Hongpaisan J, Sun MK, Alkon DL: PKC epsilon activation prevents synaptic loss, Abeta elevation, and cognitive deficits in Alzheimer's disease transgenic mice. J Neurosci 2011, 31:630-643

31. Yan J, Sun XB, Wang HQ, Zhao H, Zhao XY, Xu YX, Guo JC, Zhu CQ: Chronic restraint stress alters the expression and distribution of phosphorylated tau and MAP2 in cortex and hippocampus of rat brain. Brain Res 2010, 1347:132-141

32. Li D, Lang W, Zhou C, Wu C, Zhang F, Liu Q, Yang S, Hao J: Upregulation of microglial ZEB1 ameliorates brain damage after acute ischemic stroke. Cell Rep 2018, 22:3574-3586

33. Ding H, Zhang H, Ding H, Li D, Yi X, Ma X, Li R, Huang M, Ju X: Transplantation of placenta-derived mesenchymal stem cells reduces hypoxic-ischemic brain damage in rats by ameliorating the inflammatory response. Cell Mol Immunol 2017, 14:693-701

34. Li X, Luo S, Zhang J, Yuan Y, Jiang W, Zhu H, Ding X, Zhan L, Wu H, Xie Y, Song R, Pan Z, Lu Y: IncRNA H19 alleviated myocardial I/RI via suppressing miR-877-3p/Bcl-2-mediated mitochondrial apoptosis. Mol Ther Nucleic Acids 2019, 17:297-309

35. Yuan Y, Zheng Z: Geniposide protects PC-12 cells against oxygen and glucose deprivation-induced injury by up-regulation of longnoncoding RNA H19. Life Sci 2019, 216:176-182

36. Pan Y, Liu R, Terpstra E, Wang Y, Qiao F, Wang J, Tong Y, Pan B: Dysregulation and diagnostic potential of microRNA in Alzheimer's disease. J Alzheimers Dis 2016, 49:1-12

37. Sun X, Wei L, Chen Q, Terek RM: MicroRNA regulates vascular endothelial growth factor expression in chondrosarcoma cells. Clin Orthop Relat Res 2015, 473:907-913

38. Yang Y, Zhang X, Cui H, Zhang C, Zhu C, Li L: Apelin-13 protects the brain against ischemia/reperfusion injury through activating PI3K/Akt and ERK1/2 signaling pathways. Neurosci Lett 2014, 568: 44-49

39. Liu G, Wang T, Wang T, Song J, Zhou Z: Effects of apoptosis-related proteins caspase-3, Bax and Bcl-2 on cerebral ischemia rats. Biomed Rep 2013, 1:861-867

40. Ma Q, Dasgupta C, Li Y, Bajwa NM, Xiong F, Harding B, Hartman R, Zhang L: Inhibition of microRNA-210 provides neuroprotection in hypoxic-ischemic brain injury in neonatal rats. Neurobiol Dis 2016, 89:202-212

41. Geng JF, Liu X, Zhao HB, Fan WF, Geng JJ, Liu XZ: LncRNA UCA1 inhibits epilepsy and seizure-induced brain injury by regulating miR-495/Nrf2-ARE signal pathway. Int J Biochem Cell Biol 2018, 99:133-139

42. Feng Y, Rhodes PG, Bhatt AJ: Neuroprotective effects of vascular endothelial growth factor following hypoxic ischemic brain injury in neonatal rats. Pediatr Res 2008, 64:370-374 